\title{
ON THE CURVATURE OF METRIC CONTACT PAIRS
}

\author{
GIANLUCA BANDE, DAVID E. BLAIR, AND AMINE HADJAR
}

\begin{abstract}
We consider manifolds endowed with metric contact pairs for which the two characteristic foliations are orthogonal. We give some properties of the curvature tensor and in particular a formula for the Ricci curvature in the direction of the sum of the two Reeb vector fields. This shows that metrics associated to normal contact pairs cannot be flat. Therefore flat non-Kähler Vaisman manifolds do not exist. Furthermore we give a local classification of metric contact pair manifolds whose curvature vanishes on the vertical subbundle. As a corollary we have that flat associated metrics can only exist if the leaves of the characteristic foliations are at most three-dimensional.
\end{abstract}

\section{INTRODUCTION}

A contact pair on a smooth even-dimensional manifold $M$ is a pair of one-forms $\alpha_{1}$ and $\alpha_{2}$ of constant and complementary classes, for which $\alpha_{1}$ restricted to the leaves of the characteristic foliation of $\alpha_{2}$ is a contact form and vice versa [2, 5]. The Reeb vector fields on these contact leaves determine two global vector fields $Z_{1}$ and $Z_{2}$ called the Reeb vector fields of the pair. This notion was first introduced by Blair, Ludden and Yano [14] under the name bicontact in the context of Hermitian geometry, and further studied by Abe [1].

In [6, 8] the first and the third authors constructed metrics adapted to contact pairs as in metric contact geometry. More precisely, a metric contact pair on an even dimensional manifold is a triple $\left(\alpha_{1}, \alpha_{2}, g\right)$, where $\left(\alpha_{1}, \alpha_{2}\right)$ is a contact pair with Reeb vector fields $Z_{1}$, $Z_{2}$, and $g$ is an associated metric, i.e. a Riemannian metric such that $g\left(X, Z_{i}\right)=\alpha_{i}(X)$, for $i=1,2$, and for which the endomorphism field $\phi$, uniquely defined by $g(X, \phi Y)=$ $\left(d \alpha_{1}+d \alpha_{2}\right)(X, Y)$, satisfies

$$
\phi^{2}=-I d+\alpha_{1} \otimes Z_{1}+\alpha_{2} \otimes Z_{2} .
$$

Contact pairs always admit associated metrics for which the two characteristic foliations are orthogonal [6] or, equivalently, whose structure tensor $\phi$ is decomposable (i.e. $\phi$ preserves the characteristic distributions of $\alpha_{1}$ and $\alpha_{2}$ ).

In this paper we prove the following classification theorem which is analogous to that of the second author [12] concerning metric contact manifolds with curvature vanishing on the vertical subbundle:

Main Theorem. Let $M$ be a $(2 h+2 k+2)$-dimensional manifold endowed with a metric contact pair $\left(\alpha_{1}, \alpha_{2}, \phi, g\right)$ of type $(h, k)$ (with $\left.h \geq 1\right)$ and decomposable $\phi$. If the curvature $\mathrm{R}$

Date: December 6, 2018; MSC 2010 classification: primary 53C25; secondary 53B20, 53C12, 53B35.

The first author was supported by the Project Start-up giovani ricercatori-2009 of Università degli Studi di Cagliari and by a Visiting Professor fellowship at the Université de Haute Alsace in June 2010 and in June 2011. The second and the third authors were supported by a Visiting Professor fellowship at the Università degli Studi di Cagliari in April 2011 and January 2010 respectively, financed by Regione Autonoma della Sardegna. 
of the metric $g$ satisfies $\mathrm{R}_{X Y} Z_{i}=0(i=1,2)$, then $M$ is locally isometric to $\mathbb{E}^{h+1} \times \mathbb{S}^{h}(4) \times$ $\mathbb{E}^{k+1} \times \mathbb{S}^{k}(4)$ if $k \geq 1$ or $\mathbb{E}^{h+1} \times \mathbb{S}^{h}(4) \times \mathbb{E}^{1}$ if $k=0$.

If the manifold is complete, then its Riemannian universal covering is globally isometric to $\mathbb{E}^{h+1} \times \mathbb{S}^{h}(4) \times \mathbb{E}^{k+1} \times \mathbb{S}^{k}(4)$ if $k \geq 1$ or $\mathbb{E}^{h+1} \times \mathbb{S}^{h}(4) \times \mathbb{E}^{1}$ if $k=0$. In this statement we will understand that when $h$ (or $k$ ) is equal to 1 , the $\mathbb{S}^{h}(4)$ factor will just contribute another line to the Euclidean factor.

As a corollary we obtain that the only manifolds which can carry flat metric contact pairs are either four or six-dimensional with metric contact pairs of type $(1,0)$ or $(1,1)$ respectively. We also prove several formulae concerning the curvature tensor and the Ricci curvature of a metric associated to a contact pair with decomposable $\phi$. In particular, we show that, on a $2 n$-dimensional manifold endowed with such a structure, the Ricci curvature of the associated metric in the direction of the vector field $Z=Z_{1}+Z_{2}$ is $n-1-\frac{1}{2} \operatorname{Tr} \mathrm{h}^{2}$, where $\mathrm{h}=\frac{1}{2} \mathcal{L}_{Z} \phi$ and $\mathcal{L}_{Z}$ is the Lie derivative along $Z$.

An immediate consequence is the non-existence of flat metrics associated to normal contact pairs with decomposable endomorphism. This implies that the metric of a non-Kähler Vaisman structure on a smooth manifold cannot be flat. This is interesting since the property is local; until now this result was well known only for closed manifolds (see [19] and [16, Proposition 2.5]).

In the sequel we denote by $\Gamma(B)$ the space of sections of a vector bundle $B$, by $\operatorname{Tr}$ the trace of an endomorphism field, and by $\nabla$ the Levi-Civita connection of a given metric. All the differential objects considered are assumed to be smooth.

\section{Preliminaries ON METRIC CONTACT PAIRS}

In this section we gather the notions concerning contact pairs that will be needed in the sequel. We refer the reader to [3, 4, 5, 6, 7, 8, 9] for further informations and several examples of such structures.

2.1. Contact pairs. A pair $\left(\alpha_{1}, \alpha_{2}\right)$ of 1 -forms on a manifold is said to be a contact pair of type $(h, k)$ if (see [2, 5]):

$$
\begin{gathered}
\alpha_{1} \wedge\left(d \alpha_{1}\right)^{h} \wedge \alpha_{2} \wedge\left(d \alpha_{2}\right)^{k} \text { is a volume form, } \\
\left(d \alpha_{1}\right)^{h+1}=0 \text { and }\left(d \alpha_{2}\right)^{k+1}=0 .
\end{gathered}
$$

Since the form $\alpha_{1}$ (respectively $\alpha_{2}$ ) has constant class $2 h+1$ (respectively $2 k+1$ ), the distribution $\operatorname{Ker} \alpha_{1} \cap \operatorname{Ker} d \alpha_{1}$ (respectively $\operatorname{Ker} \alpha_{2} \cap \operatorname{Ker} d \alpha_{2}$ ) is completely integrable and then it determines the so-called characteristic foliation $\mathcal{F}_{1}$ (respectively $\mathcal{F}_{2}$ ) whose leaves are endowed with a contact form induced by $\alpha_{2}$ (respectively $\alpha_{1}$ ).

The equations

$$
\begin{gathered}
\alpha_{1}\left(Z_{1}\right)=\alpha_{2}\left(Z_{2}\right)=1, \quad \alpha_{1}\left(Z_{2}\right)=\alpha_{2}\left(Z_{1}\right)=0, \\
i_{Z_{1}} d \alpha_{1}=i_{Z_{1}} d \alpha_{2}=i_{Z_{2}} d \alpha_{1}=i_{Z_{2}} d \alpha_{2}=0,
\end{gathered}
$$

where $i_{X}$ is the contraction with the vector field $X$, determine uniquely the two vector fields $Z_{1}$ and $Z_{2}$, called Reeb vector fields. Since they commute [2, 5], they give rise to a locally free $\mathbb{R}^{2}$-action, called the Reeb action.

The tangent bundle of a manifold $M$ endowed with a contact pair can be split in different ways. For $i=1,2$, let $T \mathcal{F}_{i}$ be the subbundle determined by the characteristic foliation of $\alpha_{i}$, 
$T \mathcal{G}_{i}$ the subbundle of $T M$ whose fibers are given by ker $d \alpha_{i} \cap \operatorname{ker} \alpha_{1} \cap \operatorname{ker} \alpha_{2}$ and $\mathbb{R} Z_{1}, \mathbb{R} Z_{2}$ the line bundles determined by the Reeb vector fields. Then we have the following splittings:

$$
T M=T \mathcal{F}_{1} \oplus T \mathcal{F}_{2}=T \mathcal{G}_{1} \oplus T \mathcal{G}_{2} \oplus \mathcal{V}
$$

where $\mathcal{V}=\mathbb{R} Z_{1} \oplus \mathbb{R} Z_{2}$. Moreover we have $T \mathcal{F}_{1}=T \mathcal{G}_{1} \oplus \mathbb{R} Z_{2}$ and $T \mathcal{F}_{2}=T \mathcal{G}_{2} \oplus \mathbb{R} Z_{1}$.

Definition 2.1. We say that a vector field is vertical if it is a section of $\mathcal{V}$ and horizontal if it is a section of $T \mathcal{G}_{1} \oplus T \mathcal{G}_{2}$. The subbundles $\mathcal{V}$ and $T \mathcal{G}_{1} \oplus T \mathcal{G}_{2}$ will be called vertical and horizontal respectively.

Notice that $d \alpha_{1}$ (respectively $d \alpha_{2}$ ) is symplectic on the vector bundle $T \mathcal{G}_{2}$ (respectively $\left.T \mathcal{G}_{1}\right)$.

Example 2.2. Take $\left(\mathbb{R}^{2 h+2 k+2}, \alpha_{1}, \alpha_{2}\right)$ where $\alpha_{1}, \alpha_{2}$ are the Darboux contact forms on $\mathbb{R}^{2 h+1}$ and $\mathbb{R}^{2 k+1}$ respectively.

This is also a local model for all contact pairs of type $(h, k)$. Hence a contact pair manifold is locally the product of two contact manifolds [2, 5].

2.2. Contact pair structures. We recall now the notion of contact pair structure studied in [6, 7, 8].

Definition $2.3([6])$. A contact pair structure on a manifold $M$ is a triple $\left(\alpha_{1}, \alpha_{2}, \phi\right)$, where $\left(\alpha_{1}, \alpha_{2}\right)$ is a contact pair and $\phi$ a tensor field of type $(1,1)$ such that:

$$
\phi^{2}=-I d+\alpha_{1} \otimes Z_{1}+\alpha_{2} \otimes Z_{2}, \phi Z_{1}=\phi Z_{2}=0
$$

where $Z_{1}$ and $Z_{2}$ are the Reeb vector fields of $\left(\alpha_{1}, \alpha_{2}\right)$.

One can see that $\alpha_{i} \circ \phi=0$ for $i=1,2$ and that the rank of $\phi$ is equal to $\operatorname{dim} M-2$. Since we are also interested in the induced structures, we recall the following:

Definition 2.4 ([6]). The endomorphism $\phi$ is said to be decomposable if $\phi\left(T \mathcal{F}_{i}\right) \subset T \mathcal{F}_{i}$, for $i=1,2$.

The condition for $\phi$ to be decomposable is equivalent to $\phi\left(T \mathcal{G}_{i}\right)=T \mathcal{G}_{i}$ for $i=1,2$.

If $\phi$ is decomposable, then $\left(\alpha_{1}, Z_{1}, \phi\right)$ (respectively $\left.\left(\alpha_{2}, Z_{2}, \phi\right)\right)$ induces, on every leaf of $\mathcal{F}_{2}$ (respectively $\mathcal{F}_{1}$ ), an almost contact structure (see e.g. [13]) consisting of a contact form, its Reeb vector field and a structure tensor, the restriction of $\phi$ to the leaf.

On a manifold $M$ endowed with a contact pair, there always exists a decomposable endomorphism field $\phi$ satisfying (11) (see [6]).

As a trivial example one can take two contact manifolds $M_{i}, i=1,2$ with structure tensors $\left(\alpha_{i}, \phi_{i}\right)$, and consider the contact pair structure $\left(\alpha_{1}, \alpha_{2}, \phi_{1} \oplus \phi_{2}\right)$ on $M_{1} \times M_{2}$. In [7] we gave examples of contact pair structures with decomposable endomorphism which are not locally products.

In what follows, on a manifold $M$ endowed with a contact pair structure $\left(\alpha_{1}, \alpha_{2}, \phi\right)$, we will consider the tensor fields defined by:

$$
\begin{aligned}
N^{1}(X, Y) & =[\phi, \phi](X, Y)+2 d \alpha_{1}(X, Y) Z_{1}+2 d \alpha_{2}(X, Y) Z_{2}, \\
N_{i}^{2}(X, Y) & =\left(\mathcal{L}_{\phi X} \alpha_{i}\right)(Y)-\left(\mathcal{L}_{\phi Y} \alpha_{i}\right)(X), i=1,2, \\
\mathrm{~h} & =\frac{1}{2} \mathcal{L}_{Z} \phi
\end{aligned}
$$


for all $X, Y \in \Gamma(T M)$, where $Z=Z_{1}+Z_{2}$ and $[\phi, \phi]$ is the Nijenhuis tensor of $\phi$. The vanishing of $N^{1}$ gives exactly the normality of the pair [7], that is the integrability of the two almost complex structures $\phi \pm\left(\alpha_{1} \otimes Z_{2}-\alpha_{2} \otimes Z_{1}\right)$. In this case, by [7, Equation (3.5)] we have the following:

Proposition 2.5. If a contact pair structure $\left(\alpha_{1}, \alpha_{2}, \phi\right)$ with Reeb vector fields $Z_{1}$ and $Z_{2}$ is normal, we have $N_{1}^{2}=N_{2}^{2}=0, \mathcal{L}_{Z_{1}} \phi=\mathcal{L}_{Z_{2}} \phi=0$ and then $\mathrm{h}=0$.

2.3. Metric contact pairs. On manifolds endowed with contact pair structures it is natural to consider the following metrics:

Definition 2.6 ([6]). Let $\left(\alpha_{1}, \alpha_{2}, \phi\right)$ be a contact pair structure on a manifold $M$, with Reeb vector fields $Z_{1}$ and $Z_{2}$. A Riemannian metric $g$ on $M$ is said to be:

i) compatible if $g(\phi X, \phi Y)=g(X, Y)-\alpha_{1}(X) \alpha_{1}(Y)-\alpha_{2}(X) \alpha_{2}(Y)$ for all $X, Y \in \Gamma(T M)$,

ii) associated if $g(X, \phi Y)=\left(d \alpha_{1}+d \alpha_{2}\right)(X, Y)$ and $g\left(X, Z_{i}\right)=\alpha_{i}(X)$, for $i=1,2$ and for all $X, Y \in \Gamma(T M)$.

An associated metric is compatible, but the converse is not true.

Definition 2.7 ([6]). A metric contact pair (MCP) on a manifold $M$ is a four-tuple $\left(\alpha_{1}, \alpha_{2}, \phi, g\right)$ where $\left(\alpha_{1}, \alpha_{2}, \phi\right)$ is a contact pair structure and $g$ an associated metric with respect to it. The manifold $M$ will be called an MCP manifold or simply an $M C P$.

For an MCP $\left(\alpha_{1}, \alpha_{2}, \phi, g\right)$ the endomorphism field $\phi$ is decomposable if and only if the characteristic foliations $\mathcal{F}_{1}, \mathcal{F}_{2}$ are orthogonal [6]. In this case $\left(\alpha_{i}, \phi, g\right)$ induces a metric contact structure on the leaves of $\mathcal{F}_{j}$, for $j \neq i$.

Using a standard polarization on the symplectic vector bundles $T \mathcal{G}_{i}$, one can see that for a given contact pair $\left(\alpha_{1}, \alpha_{2}\right)$ there always exist a decomposable endomorphism field $\phi$ and a metric $g$ such that $\left(\alpha_{1}, \alpha_{2}, \phi, g\right)$ is an MCP (see [6]). Moreover we have:

Proposition 2.8. Let $\left(\alpha_{1}, \alpha_{2}, \phi, g\right)$ be an MCP with decomposable $\phi$. Then we have:

$$
N_{1}^{2}=N_{2}^{2}=0 \text {. }
$$

Proof. Since $\phi$ is decomposable, if $X, Y$ are vector fields tangent to different foliations, we have $d \alpha_{i}(\phi X, Y)=d \alpha_{i}(\phi Y, X)=0, i=1,2$. If $X, Y$ are tangent to the same foliation $\mathcal{F}_{i}$, because $\phi$ preserves the foliation, we have $N_{i}^{2}(X, Y)=0$. Moreover, for $j \neq i$, the triple $\left(\alpha_{j}, \phi, g\right)$ restricted to the leaves of $\mathcal{F}_{i}$ is a metric contact structure and then it satisfies (2), which is a well known fact in metric contact geometry.

Some other properties of MCP's are given by the following results:

Theorem $2.9([6])$. Let $M$ be a manifold endowed with a contact pair structure $\left(\alpha_{1}, \alpha_{2}, \phi\right)$, with Reeb vector fields $Z_{1}, Z_{2}$. Let $g$ be a metric compatible metric with the structure. Then we have:

(1) $g\left(Z_{i}, X\right)=\alpha_{i}(X)$ for $i=1,2$ and for every $X \in \Gamma(T M)$;

(2) $g\left(Z_{i}, Z_{j}\right)=\delta_{i j}$ for $i, j=1,2$;

(3) $\nabla_{Z_{i}} Z_{j}=0$ for $i, j=1,2$ (in particular the integral curves of the Reeb vector fields are geodesics);

(4) the Reeb action is totally geodesic (i.e. the orbits are totally geodesic two-dimensional submanifolds). 
Moreover, if $g$ is an associated metric, then $\mathcal{L}_{Z_{i}} \phi=0$ if and only if $Z_{i}$ is Killing.

In the normal case, by Proposition 2.5, an immediate consequence is:

Corollary 2.10. If an $M C P\left(\alpha_{1}, \alpha_{2}, \phi, g\right)$ is normal, the Reeb vector fields $Z_{1}$ and $Z_{2}$ are Killing.

Now using the invariance of the $\alpha_{i}$ 's under the flow of $Z=Z_{1}+Z_{2}$ one can also prove the following:

Proposition 2.11. Let $\left(\alpha_{1}, \alpha_{2}, \phi, g\right)$ be an $M C P$ with Reeb vector fields $Z_{1}$ and $Z_{2}$. Then $\mathrm{h}$ vanishes if and only if $Z$ is Killing.

We end this section with a result from [8]:

Theorem 2.12 ([8]). On an MCP manifold $\left(M, \alpha_{1}, \alpha_{2}, \phi, g\right)$ with decomposable $\phi$ the leaves of the characteristic foliations of the contact pair are orthogonal and minimal.

As example, one can simply take the product of two metric contact manifolds. Here is an interesting example from [8] which shows that an MCP manifold is not always locally the product of two metric contact manifolds:

Example 2.13. Let us consider the simply connected 6-dimensional nilpotent Lie group $G$ with structure equations:

$$
\begin{gathered}
d \omega_{3}=d \omega_{6}=0, \quad d \omega_{2}=\omega_{5} \wedge \omega_{6}, \\
d \omega_{1}=\omega_{3} \wedge \omega_{4}, d \omega_{4}=\omega_{3} \wedge \omega_{5}, \quad d \omega_{5}=\omega_{3} \wedge \omega_{6},
\end{gathered}
$$

where the $\omega_{i}$ 's form a basis for the cotangent space of $G$ at the identity. Then $\left(\omega_{1}, \omega_{2}\right)$ together with the metric

$$
g=\omega_{1}^{2}+\omega_{2}^{2}+\frac{1}{2} \sum_{i=3}^{6} \omega_{i}^{2}
$$

is a left invariant MCP of type $(1,1)$ on $G$. Note that the two characteristic foliations are orthogonal, and that their leaves, although minimal, are not totally geodesic. So the metric $g$ is not locally a product. Since the structure constants of the group are rational, there exist lattices $\Gamma$ such that $G / \Gamma$ is compact. This MCP descends to all quotients $G / \Gamma$, and we obtain closed nilmanifolds carrying the same type of structure. Moreover one can see that these MCP structures are not normal, their Reeb vector fields are however Killing and hence $\mathrm{h}=0$.

\section{The tensor h And the Levi-Civita CONNECTion For MCP's}

Here we show some properties of the tensor field $\mathrm{h}$ for MCP manifolds. We also prove some formulae concerning the Levi-Civita connection $\nabla$ for a metric associated to a contact pair.

\subsection{The covariant derivative of $\phi$.}


Proposition 3.1. Let $\left(\alpha_{1}, \alpha_{2}, \phi\right)$ be a contact pair structure together with a compatible metric $g$, and $\Phi$ the two-form defined by $\Phi(X, Y)=g(\phi X, Y)$. Then the covariant derivative of $\phi$ is given by

$$
\begin{aligned}
2 g & \left(\left(\nabla_{X} \phi\right) Y, W\right)=3 d \Phi(X, Y, W)-3 d \Phi(X, \phi Y, \phi W)+g\left(N^{1}(Y, W), \phi X\right) \\
& +2 \sum_{i=1}^{2}\left(d \alpha_{i}(\phi Y, X) \alpha_{i}(W)-d \alpha_{i}(\phi W, X) \alpha_{i}(Y)\right)+\sum_{i=1}^{2} \alpha_{i}(X) N_{i}^{2}(Y, W) .
\end{aligned}
$$

Proof. Applying the definition of the Levi-Civita connection to $2 g\left(\nabla_{X} Y, W\right)$ and using the formula for the exterior derivative of $\Phi$ in terms of Lie brackets, we have:

$$
\begin{aligned}
& 2 g\left(\left(\nabla_{X} \phi\right) Y, W\right) \\
& =2 g\left(\nabla_{X}(\phi Y), W\right)+2 g\left(\nabla_{X} Y, \phi W\right) \\
& =X \Phi(Y, W)+\phi Y\left(\Phi(X, \phi W)+\sum_{i=1}^{2} \alpha_{i}(X) \alpha_{i}(W)\right)+W \Phi(X, Y) \\
& +\Phi([X, \phi Y], \phi W)+\sum_{i=1}^{2} \alpha_{i}([X, \phi Y]) \alpha_{i}(W)-\Phi([W, X], Y)-g(\phi[\phi Y, W], \phi X) \\
& -\sum_{i=1}^{2} \alpha_{i}([\phi Y, W]) \alpha_{i}(X)-X \Phi(\phi Y, \phi W)+Y \Phi(W, X) \\
& -\phi W\left(\Phi(X, \phi Y)+\sum_{i=1}^{2} \alpha_{i}(X) \alpha_{i}(Y)\right)-\Phi([X, Y], W)+\Phi([\phi W, X], \phi Y) \\
& +\sum_{i=1}^{2} \alpha_{i}([\phi W, X]) \alpha_{i}(Y)-g(\phi[Y, \phi W], \phi X)+\sum_{i=1}^{2} \alpha_{i}(X) \alpha_{i}([\phi W, Y]) \\
& -g([Y, W], \phi X)-\Phi([Y, W], X)+g([\phi Y, \phi W], \phi X)+\Phi([\phi Y, \phi W], X) \\
& +g\left(2 d \alpha_{1}(Y, W) Z_{1}, \phi X\right)+g\left(2 d \alpha_{2}(Y, W) Z_{2}, \phi X\right) \\
& =3 d \Phi(X, Y, W)-3 d \Phi(X, \phi Y, \phi W)+g\left(N^{1}(Y, W), \phi X\right) \\
& +2 \sum_{i=1}^{2}\left(d \alpha_{i}(\phi Y, X) \alpha_{i}(W)-d \alpha_{i}(\phi W, X) \alpha_{i}(Y)\right)+\sum_{i=1}^{2} \alpha_{i}(X) N_{i}^{2}(Y, W) .
\end{aligned}
$$

Applying Proposition 3.1 to a MCP with decomposable $\phi$, we obtain:

Corollary 3.2. For an $\operatorname{MCP}\left(\alpha_{1}, \alpha_{2}, \phi, g\right)$ with decomposable $\phi$, the covariant derivative of $\phi$ is given by

(4) $2 g\left(\left(\nabla_{X} \phi\right) Y, W\right)=g\left(N^{1}(Y, W), \phi X\right)+2 \sum_{i=1}^{2}\left(d \alpha_{i}(\phi Y, X) \alpha_{i}(W)-d \alpha_{i}(\phi W, X) \alpha_{i}(Y)\right)$. 
Proof. For an MCP with decomposable $\phi$ we have $N_{1}^{2}=N_{2}^{2}=0$ by Proposition 2.8. Moreover $-\Phi=d \alpha_{1}+d \alpha_{2}$. Then (3) reduces to (41).

Corollary 3.3. For an MCP with decomposable $\phi$ and Reeb vector fields $Z_{1}, Z_{2}$ we have:

$$
\nabla_{Z_{1}} \phi=\nabla_{Z_{2}} \phi=0 \text {. }
$$

Proof. In (4), we put $X=Z_{i}$ for $i=1,2$, and we obtain $g\left(\left(\nabla_{Z_{i}} \phi\right) Y, W\right)=0$.

3.2. The tensor field $\mathrm{h}$. When $\phi$ is decomposable so is the tensor field $\mathrm{h}$, because for every $X \in \Gamma\left(T \mathcal{F}_{i}\right)$ we have $\left[Z_{j}, X\right] \in \Gamma\left(T \mathcal{F}_{i}\right)$ for $i, j=1,2$. In this case we have:

$$
\mathrm{h}=\mathrm{h}_{1} \oplus \mathrm{h}_{2} \text { and } \phi=\phi_{1} \oplus \phi_{2},
$$

where $\mathrm{h}_{1}$ (respectively $\phi_{1}$ ) is the endomorphism of $T \mathcal{F}_{2}$ induced by h (respectively by $\phi$ ) and vice-versa. We can now state the following results:

Theorem 3.4. Let $\left(\alpha_{1}, \alpha_{2}, \phi, g\right)$ be an MCP with decomposable $\phi$ on a manifold $M$. Let $Z_{1}, Z_{2}$ be the Reeb vector fields of $\left(\alpha_{1}, \alpha_{2}\right)$ and $Z=Z_{1}+Z_{2}$. Then we have:

(a) $\mathcal{L}_{Z_{1}} \phi, \mathcal{L}_{Z_{2}} \phi, \mathrm{h}, \mathrm{h}_{1}$ and $\mathrm{h}_{2}$ are symmetric operators;

(b) $\nabla_{X} Z=-\phi X-\phi \mathrm{h} X$ for every $X \in \Gamma(T M)$;

(c) $\mathrm{h} \circ \phi+\phi \circ \mathrm{h}=0$ and $\mathrm{h}_{i} \circ \phi_{i}+\phi_{i} \circ \mathrm{h}_{i}=0$ for $i=1,2$;

(d) $\operatorname{Trh}=\operatorname{Tr} \mathrm{h}_{1}=\operatorname{Tr} \mathrm{h}_{2}=0$;

(e) $\alpha_{i} \circ \mathrm{h}=\alpha_{i} \circ \mathrm{h}_{j}=0$ for every $i, j=1,2$.

To prove this we need the following:

Lemma 3.5. Let $\left(\alpha_{1}, \alpha_{2}, \phi, g\right)$ be an $M C P$ on a manifold $M$ with Reeb vector fields $Z_{1}$ and $Z_{2}$. Then for every $X \in \Gamma(T M), \nabla_{X} Z_{1}$ and $\nabla_{X} Z_{2}$ are both tangent to the kernels of $\alpha_{1}$ and $\alpha_{2}$.

Proof. Since $\nabla_{Z_{i}} Z_{j}=0$, it is enough to take $X$ horizontal. Note also that $\alpha_{1}\left(\nabla_{X} Z_{2}\right)=$ $-\alpha_{2}\left(\nabla_{X} Z_{1}\right)$ and $\alpha_{1}\left(\nabla_{X} Z_{1}\right)=\alpha_{2}\left(\nabla_{X} Z_{2}\right)=0$. Now

$$
\alpha_{1}\left(\nabla_{X} Z_{2}\right)=g\left(\nabla_{Z_{2}} X+\left[X, Z_{2}\right], Z_{1}\right)=\alpha_{1}\left(\left[X, Z_{2}\right]\right)+Z_{2} \alpha_{1}(X)-g\left(X, \nabla_{Z_{2}} Z_{1}\right)=0
$$

since $d \alpha_{1}\left(X, Z_{2}\right)=0$.

Proof of Theorem 3.4.

(a) We want to show that $g\left(X,\left(\mathcal{L}_{Z_{j}} \phi\right) Y\right)=g\left(\left(\mathcal{L}_{Z_{j}} \phi\right) X, Y\right)$, for $j=1,2$. We prove the property for $j=1$, since the other case is similar. For $X=Z_{i}, i=1,2$ we have $g\left(\left(\mathcal{L}_{Z_{1}} \phi\right) Z_{i}, Y\right)=0$ and $g\left(Z_{i},\left(\mathcal{L}_{Z_{1}} \phi\right) Y\right)=0$. The same holds for $Y=Z_{i}, i=1,2$. Then we have to prove the symmetry of $\mathcal{L}_{Z_{1}} \phi$ for $X, Y$ tangent to ker $\alpha_{1} \cap \operatorname{ker} \alpha_{2}$. By Corollary 3.3 we have $\nabla_{Z_{i}} \phi=0$ for $i=1,2$. For $X, Y \in \operatorname{ker} \alpha_{1} \cap \operatorname{ker} \alpha_{2}$, we have:

$$
\begin{aligned}
g\left(\left(\mathcal{L}_{Z_{1}} \phi\right) X, Y\right) & =g\left(-\nabla_{\phi X} Z_{1}+\phi\left(\nabla_{X} Z_{1}\right), Y\right) \\
& =g\left(Z_{1}, \nabla_{\phi X} Y\right)-g\left(\nabla_{X} Z_{1}, \phi Y\right) \\
& =\alpha_{1}\left(\nabla_{\phi X} Y\right)+\alpha_{1}\left(\nabla_{X} \phi Y\right) \\
& =\alpha_{1}\left(\nabla_{Y} \phi X\right)+\alpha_{1}\left(\nabla_{\phi Y} X\right) \\
& =g\left(X,\left(\mathcal{L}_{Z_{1}} \phi\right) Y\right),
\end{aligned}
$$


where we have used that $Z_{1}$ is orthogonal to $X, Y \in \operatorname{ker} \alpha_{1} \cap \operatorname{ker} \alpha_{2}$ and that for an MCP the tensors $N_{1}^{2}$ and $N_{2}^{2}$ vanish. It is clear that h is symmetric as well and after restriction this is also true for $h_{1}$ and $h_{2}$.

(b) By Corollary [3.2, for $i=1,2$, and for every $X, Y \in \Gamma(T M)$, we have:

$$
\begin{aligned}
2 g\left(\left(\nabla_{X} \phi\right) Z_{i}, Y\right) & =g\left(N^{1}\left(Z_{i}, Y\right), \phi X\right)-2 d \alpha_{i}(\phi Y, X) \\
& =g\left(\phi^{2}\left[Z_{i}, Y\right]-\phi\left[Z_{i}, \phi Y\right], \phi X\right)-2 d \alpha_{i}(\phi Y, X) \\
& \left.=-g\left(\phi\left(\mathcal{L}_{Z_{i}} \phi\right) Y\right), \phi X\right)-2 d \alpha_{i}(\phi Y, X) \\
& \left.=-g\left(\left(\mathcal{L}_{Z_{i}} \phi\right) Y\right), X\right)+\left(\sum_{j=1}^{2} \alpha_{j}\left(\left(\mathcal{L}_{Z_{i}} \phi\right) Y\right) \alpha_{j}(X)\right)-2 d \alpha_{i}(\phi Y, X) \\
& =-g\left(\left(\mathcal{L}_{Z_{i}} \phi\right) Y, X\right)-2 d \alpha_{i}(\phi Y, X) .
\end{aligned}
$$

Then we obtain:

$$
\begin{aligned}
\sum_{i=1}^{2} 2 g\left(\left(\nabla_{X} \phi\right) Z_{i}, Y\right) & =\sum_{i=1}^{2}\left(-2 g\left(\left(\mathcal{L}_{Z_{i}} \phi\right) Y, X\right)-2 d \alpha_{i}(\phi Y, X)\right) \\
& =-2 \sum_{i=1}^{2} g\left(\left(\mathcal{L}_{Z_{i}} \phi\right) Y, X\right)-2 g(\phi Y, \phi X) \\
& =-2 \sum_{i=1}^{2} g\left(\left(\mathcal{L}_{Z_{i}} \phi\right) Y, X\right)-2 g(Y, X)+2 \sum_{i=1}^{2} \alpha_{i}(X) \alpha_{i}(Y)
\end{aligned}
$$

and then

$$
\begin{aligned}
g\left(\left(\nabla_{X} \phi\right) Z, Y\right) & =-g(\mathrm{~h} Y, X)-g(X, Y)+\sum_{i=1}^{2} \alpha_{i}(X) \alpha_{i}(Y) \\
& =-g(\mathrm{~h} Y, X)-g(X, Y)+\sum_{i=1}^{2} g\left(\alpha_{i}(X) Z_{i}, Y\right) .
\end{aligned}
$$

Since the last equation is true for every $X, Y \in \Gamma(T M)$, this implies

$$
-\phi \nabla_{X} Z=\left(\nabla_{X} \phi\right) Z=-\mathrm{h} X-X+\alpha_{1}(X) Z_{1}+\alpha_{2}(X) Z_{2}
$$

Applying $\phi$ to the last equation and using Lemma 3.5 gives:

$$
\nabla_{X} Z=-\phi X-\phi \mathrm{h} X+\alpha_{1}\left(\nabla_{X} Z\right) Z_{1}+\alpha_{2}\left(\nabla_{X} Z\right) Z_{2}=-\phi X-\phi \mathrm{h} X
$$


(c) For $X, Y \in \Gamma(T M)$, by the symmetry of $\mathrm{h}$ and the formula $\nabla_{X} Z=-\phi X-\phi \mathrm{h} X$, we have:

$$
\begin{aligned}
2 g(X, \phi Y) & =2\left(d \alpha_{1}+d \alpha_{2}\right)(X, Y) \\
& =\sum_{i=1}^{2}\left(g\left(\nabla_{X} Z_{i}, Y\right)-g\left(\nabla_{Y} Z_{i}, X\right)\right) \\
& =g\left(\nabla_{X} Z, Y\right)-g\left(\nabla_{Y} Z, X\right) \\
& =-g(\phi X, Y)+g(\phi Y, X)-g(\phi \mathrm{h} X, Y)+g(\phi \mathrm{h} Y, X) \\
& =-g(\phi \mathrm{h} X, Y)+g(\phi \mathrm{h} Y, X)+2 g(X, \phi Y) \\
& =g(\mathrm{~h} \phi Y+\phi \mathrm{h} Y, X)+2 g(X, \phi Y),
\end{aligned}
$$

which implies that $\mathrm{h} \circ \phi+\phi \circ \mathrm{h}=0$. After restriction of $\mathrm{h}$ and $\phi$ to $T \mathcal{F}_{i}$ for $i=1,2$, we obtain $\mathrm{h}_{i} \circ \phi_{i}+\phi_{i} \circ \mathrm{h}_{i}=0$.

(d) Since the endomorphism $\mathrm{h}$ is symmetric, at every point $p \in M$ there exists an eigenbasis of $T_{p} M$. Let $V$ be an eigenvector relative to the eigenvalue $\lambda$. Then, by (c), we have:

$$
\mathrm{h}_{p}\left(\phi_{p} V\right)=-\lambda\left(\phi_{p} V\right),
$$

which means that $-\lambda$ is also an eigenvalue, relative to the eigenvector $\phi_{p} V$, and then the trace of $\mathrm{h}_{p}$ vanishes for every $p \in M$. Similarly we have $\operatorname{Tr} \mathrm{h}_{1}=\operatorname{Tr} \mathrm{h}_{2}=0$.

(e) The last property follows easily from (c).

Corollary 3.6. Let $\left(\alpha_{1}, \alpha_{2}, \phi, g\right)$ be an MCP with decomposable $\phi$ and Reeb vector fields $Z_{1}$, $Z_{2}$. If the vector field $Z=Z_{1}+Z_{2}$ is Killing, then we have

$$
\nabla_{X} Z=-\phi X \text {. }
$$

Proof. By Proposition 2.11, the vector field $Z$ is Killing if and only if $\mathrm{h}=0$. Applying this to Theorem 3.4 (b), we get $\nabla_{X} Z=-\phi X$.

A special case is given when both Reeb vector fields are Killing. A first example of the latter situation concerns the non-normal MCP on the nilpotent Lie group $G$ and its closed nilmanifolds $G / \Gamma$ described in Example 2.13,

One can also have $Z_{i}$ Killing by choosing normal structures (see Corollary 2.10). Then here is a second example, with a normal MCP but where the manifold is not a product of two metric contact manifolds:

Example 3.7. Let $M=\widetilde{S L_{2}}$ be the universal covering of the identity component of the isometry group of the hyperbolic plane $\mathbb{H}^{2}$ endowed with an invariant Sasakian structure $(\alpha, \phi, g)$ (see [17]) and $N=M \times M$. It is well known that $N$ admits cocompact irreducible lattices $\Gamma$ (see [15]). This means that $\Gamma$ does not admit any subgroup of finite index which is a product of two lattices of $M$. The manifold $N$ can be endowed with the product MCP structure and by the invariance of the structure by $\Gamma$, the MCP descends to the quotient and is normal. Even if the local structure is like a product, globally the two characteristic foliations can be very interesting in the sense that both could have dense leaves. 


\section{Some Curvature properties}

In this section, for a manifold $M$ carrying an $\operatorname{MCP}\left(\alpha_{1}, \alpha_{2}, \phi, g\right)$ with decomposable $\phi$, we set $Z=Z_{1}+Z_{2}$ where $Z_{1}, Z_{2}$ are the Reeb vector fields. We prove some properties of the curvature tensor and the Ricci curvature, which are analogous to those of metric contact structures (see e.g. [13]). As a consequence we prove the non-flatness of metrics associated to normal MCP's. This implies the non-existence of flat non-Kähler Vaisman manifolds.

4.1. The curvature. We denote by $\mathrm{R}$ the curvature tensor of the metric $g$, and by Ric its Ricci curvature.

Proposition 4.1. Let $\left(\alpha_{1}, \alpha_{2}, \phi, g\right)$ be an $M C P$ with decomposable $\phi$ on a manifold $M$. Then:

$$
\begin{aligned}
& \left(\nabla_{Z} \mathrm{~h}\right) X=\phi X-\mathrm{h}^{2} \phi X-\phi \mathrm{R}_{X Z} Z \\
& \frac{1}{2}\left(\mathrm{R}_{Z X} Z-\phi\left(\mathrm{R}_{Z \phi X} Z\right)\right)=\phi^{2} X+\mathrm{h}^{2} X .
\end{aligned}
$$

Proof. Corollary 3.3 implies $\nabla_{Z}(\phi X)=\phi\left(\nabla_{Z} X\right)$. Using this and Theorems 2.9 and 3.4, we apply $\phi$ to:

$$
R_{Z X} Z=\nabla_{Z} \nabla_{X} Z-\nabla_{X} \nabla_{Z} Z-\nabla_{[Z, X]} Z=\nabla_{Z}(-\phi X-\phi \mathrm{h} X)+\phi[Z, X]+\phi \mathrm{h}[Z, X]
$$

and we obtain:

$$
\begin{aligned}
\phi\left(R_{Z X} Z\right) & =\nabla_{Z}(X+\mathrm{h} X)-\alpha_{1}\left(\nabla_{Z}(X+\mathrm{h} X)\right) Z_{1}-\alpha_{2}\left(\nabla_{Z}(X+\mathrm{h} X)\right) Z_{2}-[Z, X] \\
& +\alpha_{1}([Z, X]) Z_{1}+\alpha_{2}([Z, X]) Z_{2}-\mathrm{h}[Z, X]+\alpha_{1}(\mathrm{~h}[Z, X]) Z_{1}+\alpha_{2}(\mathrm{~h}[Z, X]) Z_{2} \\
& =\left(\nabla_{Z} \mathrm{~h}\right) X+\nabla_{X} Z+\mathrm{h} \nabla_{X} Z \\
& =\left(\nabla_{Z} \mathrm{~h}\right) X-\phi X-\phi \mathrm{h} X+\mathrm{h}(-\phi X-\phi \mathrm{h} X) \\
& =\left(\nabla_{Z} \mathrm{~h}\right) X-\phi X+\mathrm{h}^{2} \phi X
\end{aligned}
$$

which gives (5).

To prove ([6) we first remark that $R_{Z X} Z$ is tangent to the kernels of $\alpha_{1}$ and $\alpha_{2}$. Then we have:

$$
R_{Z X} Z=-\phi^{2} R_{Z X} Z=\phi^{2} X-\phi \mathrm{h}^{2} \phi X-\phi\left(\left(\nabla_{Z} \mathrm{~h}\right) X\right)=\phi^{2} X+\mathrm{h}^{2} X-\phi\left(\left(\nabla_{Z} \mathrm{~h}\right) X\right) .
$$

Using the previous expression and taking the difference $R_{Z X} Z-\phi\left(R_{Z \phi X} Z\right.$ ) gives (6)).

Theorem 4.2. Let $\left(\alpha_{1}, \alpha_{2}, \phi, g\right)$ be an MCP of type $(h, k)$ with decomposable $\phi$ on a $(2 h+$ $2 k+2)$-dimensional manifold $M$. Then we have:

$$
\operatorname{Ric}(Z)=h+k-\frac{1}{2} \operatorname{Tr} h^{2} .
$$

Moreover $\operatorname{Ric}(Z)=h+k$ if and only if $Z$ is Killing.

Proof. Denote by $K(Z, X)$ the sectional curvature of the plane determined by $\{Z, X\}$. By using (6) for $X$ of unit length and orthogonal to $Z_{1}$ and $Z_{2}$, and recalling that $g(Z, Z)=2$, 
we obtain

$$
\begin{aligned}
\mathrm{K}(Z, X)+\mathrm{K}(Z, \phi X) & =-\frac{1}{2} g\left(\mathrm{R}_{Z X} Z-\phi\left(\mathrm{R}_{Z \phi X} Z\right), X\right) \\
& =-g\left(\phi^{2} X+\mathrm{h}^{2} X, X\right) \\
& =1-g\left(\mathrm{~h}^{2} X, X\right) .
\end{aligned}
$$

Let $\left\{Z_{1}, Z_{2}, X_{1}, \cdots, X_{2 h+2 k}\right\}$ be a local $\phi$-basis, that is an orthogonal basis for which the $X_{i}$ have unit length and $X_{2 i}=\phi X_{2 i-1}$. Then, since $K\left(Z, Z_{1}-Z_{2}\right)=0$, taking the sum $\sum_{i=1}^{2 h+2 k} \mathrm{~K}\left(Z, X_{i}\right)$ we obtain (17). Now $\operatorname{Ric}(Z)=h+k$ if and only if $\operatorname{Tr} \mathrm{h}^{2}=0$. Because $\mathrm{h}$ is symmetric the trace of $h^{2}$ vanishes if and only if $h=0$. Now use Proposition 2.11 to complete the proof.

The following result generalizes to MCP's a theorem of Hatakeyama et al. [18]:

Theorem 4.3. Let $\left(\alpha_{1}, \alpha_{2}, \phi, g\right)$ be an MCP with decomposable $\phi$ on a $(2 h+2 k+2)$ dimensional manifold $M$. Then $Z$ is Killing if and only if for all the plane sections $(Z, X)$ with $X$ orthogonal to both $Z_{1}$ and $Z_{2}$, the value of the sectional curvature $\mathrm{K}(X, Z)$ is $1 / 2$. Moreover in this case, for every $Y$ we have:

$$
\mathrm{R}_{Y Z} Z=Y-\alpha_{1}(Y) Z_{1}-\alpha_{2}(Y) Z_{2} .
$$

Proof. If for all the plane sections $(Z, X)$ with $X$ orthogonal to $Z_{1}$ and $Z_{2}$, we have $\mathrm{K}(X, Z)=$ $1 / 2$. Then $\operatorname{Ric}(Z)=h+k$, and $\mathrm{h}=0$ by Theorem 4.2 .

Conversely, let $\mathrm{h}=0$. Using $\nabla_{X} Z=-\phi X$, for $X$ of unit length and orthogonal to $Z_{1}$ and $Z_{2}$, and recalling that $\nabla_{Z} Z=0$, we have

$$
\begin{aligned}
2 \mathrm{~K}(Z, X) & =-g\left(R_{Z X} Z, X\right) \\
& =-g\left(\nabla_{Z} \nabla_{X} Z-\nabla_{[Z, X]} Z, X\right) \\
& =g\left(\nabla_{Z} \phi X-\phi[Z, X], X\right) \\
& =g\left(\phi\left(\nabla_{Z} X\right)-\phi[Z, X], X\right) \\
& =g\left(\phi\left(\nabla_{X} Z+[Z, X]\right)-\phi[Z, X], X\right) \\
& =g\left(\phi\left(\nabla_{X} Z\right), X\right) \\
& =g\left(-\phi^{2} X, X\right) \\
& =1 .
\end{aligned}
$$

To obtain (8), we have just to set $\mathrm{h}=0$ in (5), apply $\phi$ on both sides and observe that an easy computation gives $g\left(R_{Y Z} Z, Z_{i}\right)=0$.

4.2. Normal MCP's and Vaisman structures. By Proposition 2.5, for a normal contact pair the tensor $\mathrm{h}$ vanishes necessarily. Thus, by (7), we have:

Corollary 4.4. A metric associated to a normal contact pair with decomposable endomorphism cannot be flat.

In particular this is true for normal MCP's of type $(h, 0)$ which are nothing but non-Kähler Vaisman structures modulo constant rescaling of the metric [10]. For this case the previous Corollary can be stated as: 
Theorem 4.5. The metric of a non-Kähler Vaisman manifold cannot be flat.

Here compactness is not needed. However this result was known for closed manifolds. Indeed a Vaisman structure is a particular locally conformally Kähler (lcK) structure. According to [19] (see also [16, Proposition 2.5]) a closed lcK manifold of constant curvature is necessary Kähler. Hence flat non-Kähler Vaisman structures do not exist on closed manifolds.

4.3. In complete analogy to the case of contact metric manifolds, we want to define two tensor fields that are useful for the calculations in the problem of finding metric contact pairs with curvature vanishing on the vertical subbundle. First observe that for a metric contact pair with decomposable $\phi$, we have:

$$
\begin{aligned}
& 2 g\left(\left(\nabla_{X} \phi\right) W, \phi Y\right)-2 g\left(\left(\nabla_{X} \phi\right) \phi W, Y\right) \\
= & g\left(N^{1}(W, \phi Y)-N^{1}(\phi W, Y), \phi X\right)-2 d \alpha_{1}\left(\phi^{2} Y, X\right) \alpha_{1}(W)-2 d \alpha_{2}\left(\phi^{2} Y, X\right) \alpha_{2}(W) \\
& -2 d \alpha_{1}\left(\phi^{2} W, X\right) \alpha_{1}(Y)-2 d \alpha_{2}\left(\phi^{2} W, X\right) \alpha_{2}(Y) \\
= & \alpha_{1}(Y) g\left(\left[\phi W, Z_{1}\right]-\phi\left[W, Z_{1}\right], \phi X\right)+\alpha_{2}(Y) g\left(\left[\phi W, Z_{2}\right]-\phi\left[W, Z_{2}\right], \phi X\right) \\
& +\alpha_{1}(W) g\left(\left[\phi Y, Z_{1}\right]-\phi\left[Y, Z_{1}\right], \phi X\right)+\alpha_{2}(W) g\left(\left[\phi Y, Z_{2}\right]-\phi\left[Y, Z_{2}\right], \phi X\right) \\
& +2 d \alpha_{1}(Y, X) \alpha_{1}(W)+2 d \alpha_{2}(Y, X) \alpha_{2}(W)+2 d \alpha_{1}(W, X) \alpha_{1}(Y)+2 d \alpha_{2}(W, X) \alpha_{2}(Y) .
\end{aligned}
$$

Replacing $W$ with $\phi W$ in (9), we obtain the following:

$$
\begin{aligned}
& 2 g\left(\left(\nabla_{X} \phi\right) \phi W, \phi Y\right)+2 g\left(\left(\nabla_{X} \phi\right) W, Y\right)-2 g\left(Y,\left(\nabla_{X} \phi\right)\left(\alpha_{1}(W) Z_{1}+\alpha_{2}(W) Z_{2}\right)\right) \\
= & \alpha_{1}(Y) g\left(-\left[W, Z_{1}\right]-\phi\left[\phi W, Z_{1}\right], \phi X\right)+\alpha_{2}(Y) g\left(-\left[W, Z_{2}\right]-\phi\left[\phi W, Z_{2}\right], \phi X\right) \\
& +2 d \alpha_{1}(\phi W, X) \alpha_{1}(Y)+2 d \alpha_{2}(\phi W, X) \alpha_{2}(Y) .
\end{aligned}
$$

Taking $X, Y, W$ horizontal in (9) and in (10), we have:

Using (4) with $X, Y, W$ horizontal we get

$$
\begin{gathered}
g\left(\left(\nabla_{X} \phi\right) W, \phi Y\right)=g\left(\left(\nabla_{X} \phi\right) \phi W, Y\right) . \\
g\left(\left(\nabla_{X} \phi\right) \phi W, \phi Y\right)=-g\left(\left(\nabla_{X} \phi\right) W, Y\right) .
\end{gathered}
$$

For the curvature operator we have:

$$
\begin{aligned}
\mathrm{R}_{X Y} Z & =-\nabla_{X}(\phi Y+\phi \mathrm{h} Y)+\nabla_{Y}(\phi X+\phi \mathrm{h} X)+\phi[X, Y]+\phi \mathrm{h}[X, Y] \\
& =-\left(\nabla_{X} \phi\right) Y+\left(\nabla_{Y} \phi\right) X-\left(\nabla_{X} \phi \mathrm{h}\right) Y+\left(\nabla_{X} \phi \mathrm{h}\right) Y,
\end{aligned}
$$

which gives:

$$
g\left(\mathrm{R}_{Z W} X, Y\right)=-g\left(X,\left(\nabla_{W} \phi\right) Y\right)-g\left(W,\left(\nabla_{X} \phi \mathrm{h}\right) Y\right)+g\left(W,\left(\nabla_{Y} \phi \mathrm{h}\right) X\right),
$$

or equivalently

$$
g\left(\mathrm{R}_{Z X} Y, W\right)=-g\left(Y,\left(\nabla_{X} \phi\right) W\right)-g\left(X,\left(\nabla_{Y} \phi \mathrm{h}\right) W\right)+g\left(X,\left(\nabla_{W} \phi \mathrm{h}\right) Y\right) .
$$

Now we define the following tensors: 
Definition 4.6. For $X, Y, W \in \Gamma(T M)$, set

$$
\begin{gathered}
A(X, Y, W)=-g\left(Y,\left(\nabla_{X} \phi\right) W\right)+g\left(\phi Y,\left(\nabla_{X} \phi\right) \phi W\right) \\
-g\left(Y,\left(\nabla_{\phi X} \phi\right) \phi W\right)-g\left(\phi Y,\left(\nabla_{\phi X} \phi\right) W\right), \\
B(X, Y, W)=-g\left(X,\left(\nabla_{Y} \phi \mathrm{h}\right) W\right)+g\left(X,\left(\nabla_{\phi Y} \phi \mathrm{h}\right) \phi W\right) \\
-g\left(\phi X,\left(\nabla_{Y} \phi \mathrm{h}\right) \phi W\right)-g\left(\phi X,\left(\nabla_{\phi Y} \phi \mathrm{h}\right) W\right) .
\end{gathered}
$$

Taking $X, Y, W$ horizontal and using (16), we obtain the following relation:

$$
\begin{aligned}
A(X, Y, W)+B(X, Y, W)-B(X, W, Y)= & g\left(\mathrm{R}_{Z X} Y, W\right)-g\left(\mathrm{R}_{Z X} \phi Y, \phi W\right) \\
& +g\left(\mathrm{R}_{Z \phi X} Y, \phi W\right)+g\left(\mathrm{R}_{Z \phi X} \phi Y, W\right) .
\end{aligned}
$$

The following lemma will be useful in the sequel:

Lemma 4.7. For every $X, Y, W$ horizontal, we have:

$$
A(X, Y, W)+B(X, Y, W)-B(X, W, Y)=-2 g\left(\left(\nabla_{\mathrm{h} X} \phi\right) Y, W\right)
$$

Proof. For $X, Y, W$ horizontal, using (11), (12) and (13) we have:

$$
A(X, Y, W)=-2 g\left(Y,\left(\nabla_{X} \phi\right) W\right)-2 g\left(Y,\left(\nabla_{\phi X} \phi\right) \phi W\right)=0 .
$$

Also for $X, Y, W$ horizontal, we calculate

$$
\begin{aligned}
B(X, Y, W)= & -g\left(X,\left(\nabla_{Y} \phi\right) \mathrm{h} W+\phi\left(\nabla_{Y} \mathrm{~h}\right) W\right)+g\left(X,\left(\nabla_{\phi Y} \mathrm{~h}\right) W-\phi \mathrm{h}\left(\nabla_{\phi Y} \phi\right) W\right) \\
& -g\left(\phi X,\left(\nabla_{Y} \mathrm{~h}\right) W-\phi \mathrm{h}\left(\nabla_{Y} \phi\right) W\right)-g\left(\phi X,\left(\nabla_{\phi Y}\right) \mathrm{h} W+\phi\left(\nabla_{\phi Y} \mathrm{~h}\right) W\right) \\
= & -g\left(X,\left(\nabla_{Y} \phi\right) \mathrm{h} W\right)+g\left(X, \mathrm{~h} \phi\left(\nabla_{\phi Y} \phi\right) W\right) \\
& +g\left(\phi X, \phi \mathrm{h}\left(\nabla_{Y} \phi\right) W\right)-g\left(\phi X,\left(\nabla_{\phi Y} \phi\right) \mathrm{h} W\right) .
\end{aligned}
$$

Now, we have

$$
\begin{aligned}
-g\left(\phi \mathrm{h} X,\left(\nabla_{\phi Y} \phi\right) W\right) & =g\left(\left(\nabla_{\phi Y} \phi\right) \phi \mathrm{h} X, W\right) \\
& =-g\left(\left(\nabla_{Y} \phi\right) \mathrm{h} X, W\right) \\
& =g\left(\mathrm{~h} X,\left(\nabla_{Y} \phi\right) W\right),
\end{aligned}
$$

where in the second line we used (13), and furthermore we also have

$$
\begin{aligned}
-g\left(\phi X,\left(\nabla_{\phi Y} \phi\right) \mathrm{h} W\right) & =g\left(\left(\nabla_{\phi Y} \phi\right) \phi X, \mathrm{~h} W\right) \\
& =-g\left(\left(\nabla_{Y} \phi\right) X, \mathrm{~h} W\right) \\
& =g\left(X,\left(\nabla_{Y} \phi\right) \mathrm{h} W\right),
\end{aligned}
$$

again using (13). This in turn gives

$$
B(X, Y, W)=2 g\left(\mathrm{~h} X,\left(\nabla_{Y} \phi\right) W\right),
$$

and putting all this together, we obtain

$$
\begin{aligned}
A(X, Y, W)+B(X, Y, W)-B(X, W, Y) & =2 g\left(\mathrm{~h} X,\left(\nabla_{Y} \phi\right) W\right)-2 g\left(\mathrm{~h} X,\left(\nabla_{W} \phi\right) Y\right) \\
& =-2 g\left(\left(\nabla_{\mathrm{h} X} \phi\right) Y, W\right) .
\end{aligned}
$$


Corollary 4.8. If the curvature of an $M C P$ with decomposable $\phi$ satisfies $\mathrm{R}_{X Y} Z_{1}=\mathrm{R}_{X Y} Z_{2}=$ 0 for all $X, Y$, then for all horizontal vector fields

$$
g\left(\left(\nabla_{\mathrm{h} X} \phi\right) Y, W\right)=0 .
$$

Proof. The left hand side of the equation of Lemma 4.7 vanishes by the assumption on the curvature and by (19).

\section{Curvature vanishing on the Vertical subbundle}

In this section we prove for MCP's the analogues of the results of the second named author on metric contact manifolds [11, 12]. Recall that for a contact pair we defined the vertical subbundle as the subbundle $\mathcal{V}$ spanned by the Reeb vector fields $Z_{1}, Z_{2}$. We will say that an MCP has curvature vanishing on the vertical subbundle if the following condition is satisfied for all vector fields $X, Y$ :

$$
\mathrm{R}_{X Y} Z_{i}=0 \text {, for } i=1,2 .
$$

The standard example of such a situation is the product of the unit tangent bundles of $\mathbb{E}^{h}$ and $\mathbb{E}^{k}$, since each of them is endowed with a metric contact structure with curvature vanishing along the corresponding Reeb vector field (see [12]). Actually Theorem 5.1 below says exactly that locally this is the only possibility.

Before the statement of the theorem, we make some remarks. First, observe that a contact pair of type $(0,0)$ is in some sense trivial, because we would like to have an induced contact form on the leaves of at least one of the characteristic foliations. Moreover, if the manifold is endowed with an associated metric then it is flat and then locally isometric to $\mathbb{E}^{2}$. Since the forms composing the contact pair play a symmetric role, to exclude the former trivial case we only consider contact pairs of type $(h, k)$ with $h \geq 1$.

Theorem 5.1. Let $M$ be a $(2 h+2 k+2)$-dimensional manifold endowed with a metric contact pair $\left(\alpha_{1}, \alpha_{2}, \phi, g\right)$ of type $(h, k)$ (with $\left.h \geq 1\right)$ and decomposable $\phi$. If the curvature of $g$ vanishes on the vertical subbundle, then $M$ is locally isometric to $\mathbb{E}^{h+1} \times \mathbb{S}^{h}(4) \times \mathbb{E}^{k+1} \times \mathbb{S}^{k}(4)$ if $k \geq 1$ or to $\mathbb{E}^{h+1} \times \mathbb{S}^{h}(4) \times \mathbb{E}^{1}$ if $k=0$.

Proof. We split the proof into several steps:

a) We have seen that the decomposability of $\phi$ implies that $\mathrm{h}$ is also decomposable and we set $\phi=\phi_{1} \oplus \phi_{2}$ and $\mathrm{h}=\mathrm{h}_{1} \oplus \mathrm{h}_{2}$ as in Section 3.2. If the curvature tensor $\mathrm{R}$ vanishes on the vertical subbundle, by (6) we have $\mathrm{h}^{2}=-\phi^{2}$ and then, since $\mathrm{h}$ is symmetric, for its rank we have $\mathrm{rkh}=\mathrm{rkh}^{2}=\mathrm{rk} \phi^{2}=\mathrm{rk} \phi=2 h+2 k$. If $X$ is an eigenvector of h corresponding to a non-zero eigenvalue $\lambda$ then it is orthogonal to the Reeb vector fields (which are 0-eigenvectors) and we have:

$$
\lambda^{2} g(X, X)=g(\lambda X, \lambda X)=g(\mathrm{~h} X, \mathrm{~h} X)=g\left(\mathrm{~h}^{2} X, X\right)=-g\left(\phi^{2} X, X\right)=g(X, X) .
$$

Thus the non-zero eigenvalues of $h$ are \pm 1 . By restriction the same is true for the eigenvalues of $h_{1}$ and $h_{2}$. Moreover the $( \pm 1)$-eigenspaces of $h$ are direct sums of the $( \pm 1)$-eigenspaces of $\mathrm{h}_{1}$ and $\mathrm{h}_{2}$ at every point. Also observe that $\phi$ (respectively $\phi_{j}$ ) intertwines the eigenspaces corresponding to +1 and -1 , because it anticommutes with h (respectively $\mathrm{h}_{j}$ ).

Let $[-1]_{1},[-1]_{2},[-1]$ (respectively $\left.[+1]_{1},[+1]_{2},[+1]\right)$ be the $(-1)$-eigendistributions (respectively (+1)-eigendistributions) of $h_{1}, h_{2}$ and $h$ respectively. By the previous discussion we have $[-1]=[-1]_{1} \oplus[-1]_{2}$ and $[+1]=[+1]_{1} \oplus[+1]_{2}$. 
By Theorem 3.4, we obtain

$$
\nabla_{X} Z=0, \forall X \in[-1]
$$

Moreover, the vanishing of $\mathrm{R}$ on the vertical subbundle implies that for every vector fields $X, Y \in[-1]$ (respectively $[-1]_{j}$ ) we have:

$$
0=\mathrm{R}_{X Y} Z=-\nabla_{[X, Y]} Z=\phi[X, Y]+\phi \mathrm{h}[X, Y]
$$

which implies $\mathrm{h} \phi[X, Y]=\phi[X, Y]$ and then $\phi[X, Y] \in[+1]$ (respectively $[+1]_{j}$ ). Applying $\phi$ to both sides of the equation gives $\phi^{2}[X, Y] \in[-1]$ (respectively $[-1]_{j}$ ). Calculating further we have

$$
\phi^{2}[X, Y]=-[X, Y]+d \alpha_{1}(X, Y) Z_{1}+d \alpha_{2}(X, Y) Z_{2}=-[X, Y] .
$$

The last equation is clear if $X, Y$ are tangent to different foliations, since in this case the $d \alpha_{i}$ vanish, and is easily deduced from the following observation when $X, Y$ are tangent to the same foliation. In fact if $X, Y$ are tangent to the same foliation, say $\mathcal{F}_{1}$ for example, we have $d \alpha_{2}(X, Y)=d \alpha_{1}(X, Y)+d \alpha_{2}(X, Y)=g(X, \phi Y)=0$ since $X \in[-1]$ and $\phi$ intertwines the $( \pm 1)$-eigenspaces.

The same calculations give $\left[X, Z_{i}\right] \in[-1]$ (respectively $[-1]_{j}$ ) for every $X \in[-1]$ (respectively $[-1]_{j}$ ) and $\left[\phi Y, Z_{i}\right] \in[-1]$ (respectively $[-1]_{j}$ ) for every $Y \in[+1]$ (respectively $\left.[+1]_{j}\right)$. In particular this implies that the distributions $[-1]_{j},[-1],[-1]_{j} \oplus \mathbb{R} Z_{i},[-1] \oplus \mathbb{R} Z_{i}$, $[-1]_{j} \oplus \mathbb{R} Z_{1} \oplus \mathbb{R} Z_{2}$ and $[-1] \oplus \mathbb{R} Z_{1} \oplus \mathbb{R} Z_{2}$ are integrable.

b) According to the local model for contact pairs of type $(h, k)$, on every point there exist local coordinates $\left(u_{0}, \cdots, u_{2 k}, v_{0}, \cdots, v_{2 h}\right)$ such that $\frac{\partial}{\partial u_{0}}, \cdots, \frac{\partial}{\partial u_{2 k}}$ span Ker $\alpha_{1} \cap \operatorname{Ker} d \alpha_{1}$ and $\frac{\partial}{\partial v_{0}}, \cdots, \frac{\partial}{\partial v_{2 h}}$ span Ker $\alpha_{2} \cap \operatorname{Ker} d \alpha_{2}$. By the integrability of $[-1]_{2} \oplus \mathbb{R} Z_{2}$ (respectively $\left.[-1]_{1} \oplus \mathbb{R} Z_{1}\right)$, these local coordinates can be chosen such that one also has that $\frac{\partial}{\partial u_{0}}, \cdots, \frac{\partial}{\partial u_{k}}$ $\operatorname{span}[-1]_{2} \oplus \mathbb{R} Z_{2}$ and $\frac{\partial}{\partial v_{0}}, \cdots, \frac{\partial}{\partial v_{h}} \operatorname{span}[-1]_{1} \oplus \mathbb{R} Z_{1}$.

Let us define the vector fields $X_{i}=\frac{\partial}{\partial u_{k+i}}+\sum_{p=0}^{k} f_{i p} \frac{\partial}{\partial u_{p}}$, for $1 \leq i \leq k$ and $Y_{s}=\frac{\partial}{\partial v_{h+s}}+$ $\sum_{q=0}^{h} \tilde{f}_{s q} \frac{\partial}{\partial v_{q}}$ for $1 \leq s \leq h$, where the functions $f_{i p}, \tilde{f}_{s q}$ are chosen in such a way that $X_{i} \in[+1]_{2}$ and $Y_{s} \in[+1]_{1}$. In general those functions depend on all coordinates. It is clear that at every point of $M$, the $X_{i}$ 's and $Y_{s}$ 's form a basis for $[+1]_{2}$ and $[+1]_{1}$ respectively.

A direct calculation with local coordinates gives:

$$
\begin{aligned}
& {\left[\frac{\partial}{\partial u_{p}}, X_{i}\right] \in[-1]_{2} \oplus \mathbb{R} Z_{2}, 0 \leq p \leq k, 0 \leq i \leq k,} \\
& {\left[\frac{\partial}{\partial v_{q}}, Y_{s}\right] \in[-1]_{1} \oplus \mathbb{R} Z_{1}, 0 \leq q \leq h, 0 \leq s \leq h,} \\
& {\left[\frac{\partial}{\partial u_{p}}, Y_{s}\right] \in[-1]_{1} \oplus \mathbb{R} Z_{1}, 0 \leq p \leq k, 0 \leq s \leq h,} \\
& {\left[\frac{\partial}{\partial v_{q}}, X_{i}\right] \in[-1]_{2} \oplus \mathbb{R} Z_{2}, 0 \leq q \leq h, 0 \leq i \leq k,}
\end{aligned}
$$

and

$$
\left[X_{i}, Y_{s}\right] \in[-1] \oplus \mathbb{R} Z_{1} \oplus \mathbb{R} Z_{2}
$$

Then we have

$$
\nabla_{\left[\frac{\partial}{\partial u_{p}}, X_{i}\right]} Z=\nabla_{\left[\frac{\partial}{\partial u_{p}}, Y_{s}\right]} Z=\nabla_{\left[\frac{\partial}{\partial v_{q}}, X_{i}\right]} Z=\nabla_{\left[\frac{\partial}{\partial v_{q}}, Y_{s}\right]} Z=\nabla_{\left[X_{i}, Y_{s}\right]} Z=0 .
$$


The assumption on the curvature implies $\mathrm{R}_{\frac{\partial}{\partial u_{p}} X_{i}} Z=0,0 \leq p \leq k$, and we obtain

$$
0=\nabla_{\frac{\partial}{\partial u_{p}}} \nabla_{X_{i}} Z-\nabla_{X_{i}} \nabla_{\frac{\partial}{\partial u_{p}}} Z=-2 \nabla_{\frac{\partial}{\partial u_{p}}} \phi X_{i} .
$$

Since the $\frac{\partial}{\partial u_{p}}$ 's span $[-1]_{2} \oplus \mathbb{R} Z_{2}$ and the connection is tensorial in the first argument, we have:

$$
\nabla_{\phi X_{j}} \phi X_{i}=0, \forall i, j .
$$

In a similar way we obtain the following formulae:

$$
\begin{aligned}
& \nabla_{\phi Y_{r}} \phi Y_{s}=0, \forall r, s, \\
& \nabla_{\phi X_{i}} \phi Y_{r}=0, \forall i, r, \\
& \nabla_{\phi Y_{r}} \phi X_{i}=0, \forall i, r .
\end{aligned}
$$

These imply that the integral submanifolds of $[-1] \oplus \mathbb{R} Z_{1} \oplus \mathbb{R} Z_{2}$ are totally geodesic and flat.

A direct calculation with local coordinates shows that $\left[X_{i}, X_{j}\right]$ is in $[-1]_{2} \oplus \mathbb{R} Z_{2}$. Differentiating $g\left(X_{i}, Z\right)=0$ along $X_{j}$ we obtain $g\left(\nabla_{X_{j}} X_{i}, Z\right)=0$. Interchanging $i$ and $j$ and taking the difference we get $0=g\left(\left[X_{i}, X_{j}\right], Z\right)=g\left(\left[X_{i}, X_{j}\right], Z_{2}\right)$ since $\left[X_{i}, X_{j}\right]$ is orthogonal to $Z_{1}$. This actually means that $\left[X_{i}, X_{j}\right]$ is in $[-1]_{2}$.

Then we have

$$
0=\mathrm{R}_{X_{i} X_{j}} Z=-2 \nabla_{X_{i}} \phi X_{j}+2 \nabla_{X_{j}} \phi X_{i},
$$

or equivalently

$$
\nabla_{X_{i}} \phi X_{j}=\nabla_{X_{j}} \phi X_{i}
$$

Similarly we obtain

$$
\nabla_{Y_{r}} \phi Y_{s}=\nabla_{Y_{s}} \phi Y_{r}
$$

With similar calculations, using the fact that $\left[X_{i}, Y_{r}\right] \in[-1] \oplus \mathbb{R} Z_{1} \oplus \mathbb{R} Z_{2}$, we obtain

$$
\nabla_{X_{i}} \phi Y_{r}=\nabla_{Y_{r}} \phi X_{i} .
$$

Equations (29)-(31) can also be written as follows:

$$
\begin{aligned}
\phi\left[X_{i}, X_{j}\right] & =-\left(\nabla_{X_{i}} \phi\right) X_{j}+\left(\nabla_{X_{j}} \phi\right) X_{i}, \\
\phi\left[Y_{r}, Y_{s}\right] & =-\left(\nabla_{Y_{r}} \phi\right) Y_{s}+\left(\nabla_{Y_{s}} \phi\right) Y_{r}, \\
\phi\left[X_{i}, Y_{r}\right] & =-\left(\nabla_{X_{i}} \phi\right) Y_{r}+\left(\nabla_{Y_{r}} \phi\right) X_{i} .
\end{aligned}
$$

Using (25) and (27) we obtain

$$
0=\mathrm{R}_{X_{i} \phi X_{j}} Z=-\nabla_{\left[X_{i}, \phi X_{j}\right]} Z
$$

or equivalently

$$
\phi\left[X_{i}, \phi X_{j}\right]+\phi \mathrm{h}\left[X_{i}, \phi X_{j}\right]=0 .
$$

Applying $\phi$ and recalling that $\mathrm{h}\left[X_{i}, \phi X_{j}\right]$ is in the kernels of $\alpha_{1}$ and $\alpha_{2}$, we get

$$
-\left[X_{i}, \phi X_{j}\right]+\mu Z_{1}+\nu Z_{2}=\mathrm{h}\left[X_{i}, \phi X_{j}\right]
$$

for some functions $\mu, \nu$.

Taking the scalar product with $X_{l}$ and using the symmetry of h, we obtain

$$
-g\left(\left[X_{i}, \phi X_{j}\right], X_{l}\right)=g\left(\mathrm{~h}\left[X_{i}, \phi X_{j}\right], X_{l}\right)=g\left(\left[X_{i}, \phi X_{j}\right], \mathrm{h} X_{l}\right)=g\left(\left[X_{i}, \phi X_{j}\right], X_{l}\right),
$$


and finally

$$
g\left(\left[X_{i}, \phi X_{j}\right], X_{l}\right)=0
$$

Similarly we have

$$
\begin{aligned}
& g\left(\left[X_{i}, \phi X_{j}\right], Y_{s}\right)=g\left(\left[Y_{r}, \phi Y_{s}\right], X_{l}\right)=0, \\
& g\left(\left[Y_{r}, \phi X_{j}\right], X_{i}\right)=g\left(\left[Y_{r}, \phi X_{j}\right], Y_{s}\right)=0, \\
& g\left(\left[\phi Y_{r}, X_{i}\right], X_{l}\right)=g\left(\left[Y_{r}, \phi Y_{s}\right], Y_{t}\right)=g\left(\left[X_{i}, \phi Y_{r}\right], Y_{s}\right)=0 .
\end{aligned}
$$

c) Now we want to show that $\nabla_{X_{i}} X_{j} \in[+1] \oplus \mathbb{R} Z_{1} \oplus \mathbb{R} Z_{2}$. In fact, using Corollary 4.8 and (27) we have

$$
\begin{aligned}
0 & =g\left(\left(\nabla_{\phi X_{i}} \phi\right) X_{j}, \phi X_{l}\right)=-g\left(\phi\left(\nabla_{\phi X_{i}} X_{j}\right), \phi X_{l}\right) \\
& =-g\left(\nabla_{\phi X_{i}} X_{j}, X_{l}\right),
\end{aligned}
$$

where we have used the fact that $X_{l} \in \operatorname{ker} \alpha_{1} \cap \operatorname{ker} \alpha_{2}$. Since $g\left(X_{j}, \phi X_{l}\right)=0$, we obtain:

$$
\begin{aligned}
g\left(\nabla_{X_{i}} X_{j}, \phi X_{l}\right) & =-g\left(X_{j}, \nabla_{X_{i}} \phi X_{l}\right) \\
& =-g\left(X_{j}, \nabla_{\phi X_{l}} X_{i}+\left[X_{i}, \phi X_{l}\right]\right) \\
& =-g\left(X_{j},\left[X_{i}, \phi X_{l}\right]\right) \\
& =0
\end{aligned}
$$

where in the last equality we have used (33).

Similarly we obtain $g\left(\nabla_{\phi Y_{r}} X_{i}, X_{j}\right)=0$ and in turn $g\left(\nabla_{X_{i}} X_{j}, \phi Y_{r}\right)=0$. Therefore $\nabla_{X_{i}} X_{j} \in$ $[+1] \oplus \mathbb{R} Z_{1} \oplus \mathbb{R} Z_{2}$ as desired.

d) We want to show that $[+1]$ is integrable. For $X, Y, W \in[+1]$ we have:

$$
\begin{aligned}
0 & =R_{X Y} Z=\nabla_{X}(-2 \phi Y)+\nabla_{Y}(2 \phi X)+\phi[X, Y]+\phi \mathrm{h}[X, Y] \\
& =-2\left(\nabla_{X} \phi\right) Y+2\left(\nabla_{Y} \phi\right) X-\phi[X, Y]-\mathrm{h} \phi[X, Y] .
\end{aligned}
$$

Taking the inner product with $W$ and noting that $\phi W$ is in $[-1]$, we obtain

$$
0=g(\phi W,[X, Y]),
$$

which means that $[X, Y]$ is orthogonal to $[-1]$. Now let $Y \in[+1]$ and $X \in[-1]$. By the integrability of $[-1]$, we have:

$$
\begin{aligned}
0 & =d \alpha_{i}(X, \phi Y) \\
& =-d \alpha_{i}(\phi X, Y) \\
& =\frac{1}{2} \alpha_{i}([\phi X, Y]) .
\end{aligned}
$$

Then the bracket of two vector fields in $[+1]$ is also orthogonal to the vertical subbundle and this in turn implies that $[+1]$ is integrable.

e) Now we prove that $[+1]$ is totally geodesic. To show this, we will prove that, for every $Y \in[+1]$ and every $X$ orthogonal to $[+1], \nabla_{Y} X$ is orthogonal to $[+1]$.

Let us start with $Y \in[+1]$ and $X \in[-1]$ and compute

$$
\begin{aligned}
0 & =R_{X Y} Z=-2 \nabla_{X} \phi Y+\phi[X, Y]+\phi \mathrm{h}[X, Y] \\
& =-2\left(\nabla_{X} \phi\right) Y-\phi \nabla_{X} Y-\phi \nabla_{Y} X-\mathrm{h} \phi \nabla_{X} Y+\mathrm{h} \phi \nabla_{Y} X .
\end{aligned}
$$


Taking the scalar product with $W \in[-1]$, we obtain

$$
\begin{aligned}
0 & =-2 g\left(\left(\nabla_{X} \phi\right) Y, W\right)-g\left(\phi \nabla_{X} Y, W\right)-g\left(\phi \nabla_{Y} X, W\right)-g\left(\phi \nabla_{X} Y, \mathrm{~h} W\right)+g\left(\phi \nabla_{Y} X, \mathrm{~h} W\right) \\
& =-2 g\left(\left(\nabla_{X} \phi\right) Y, W\right)-2 g\left(\phi \nabla_{Y} X, W\right),
\end{aligned}
$$

which implies

$$
g\left(\left(\nabla_{X} \phi\right) Y, W\right)=-g\left(\phi \nabla_{Y} X, W\right)=g\left(\nabla_{Y} X, \phi W\right) .
$$

Since $g\left(\left(\nabla_{X} \phi\right) Y, W\right)=0$ by Corollary 4.8, we have $\nabla_{Y} X \in[-1] \oplus \mathcal{V}$.

Next observe that for $X, Y$ horizontal, we have:

$$
\begin{aligned}
2 g\left(\left(\nabla_{X} \phi\right) Z_{i}, Y\right) & =g\left(N^{1}\left(Z_{i}, Y\right), \phi X\right)-2 d \alpha_{i}(\phi Y, X) \\
& =g\left(\phi^{2}\left[Z_{i}, Y\right]-\phi\left[Z_{i}, \phi Y\right], \phi X\right)-2 d \alpha_{i}(\phi Y, X) \\
& =g\left(-\phi\left(\mathcal{L}_{Z_{i}} \phi\right) Y, \phi X\right)-2 d \alpha_{i}(\phi Y, X) \\
& =-g\left(\left(\mathcal{L}_{Z_{i}} \phi\right) Y, X\right)-2 d \alpha_{i}(\phi Y, X),
\end{aligned}
$$

then $g\left(\left(\nabla_{X} \phi\right) Z_{i}, Y\right)$ is symmetric in $X$ and $Y$.

Now for $Y \in[+1], X \in[-1]$, taking the scalar product of (38) with $Z_{i}(i=1,2)$, we get:

$$
\begin{aligned}
0 & =g\left(\left(\nabla_{X} \phi\right) Y, Z_{i}\right)=g\left(\left(\nabla_{Y} \phi\right) X, Z_{i}\right) \\
& =-g\left(\left(\nabla_{Y} \phi\right) Z_{i}, X\right)=g\left(\phi \nabla_{Y} Z_{i}, X\right) \\
& =-g\left(\left(\nabla_{Y} Z_{i}, \phi X\right),\right.
\end{aligned}
$$

which means that $\nabla_{Y} Z_{i}$ is orthogonal to $[+1]$.

This implies that $[+1]$ is totally geodesic and then, since $[-1] \oplus \mathbb{R} Z_{1} \oplus \mathbb{R} Z_{2}$ is integrable with totally geodesic leaves, the manifold splits as a local Riemannian product.

f) Using equation (4) and the integrability of $[-1] \oplus \mathbb{R} Z_{1} \oplus \mathbb{R} Z_{2}$ with totally geodesic leaves we have

$$
\begin{aligned}
2 g\left(\left(\nabla_{X_{i}} \phi\right) X_{j}, Z_{2}\right) & =g\left(\left[Z_{2}, \phi X_{j}\right]-\phi\left[Z_{2}, X_{j}\right], X_{i}\right)+2 g\left(X_{j}, X_{i}\right) \\
& =g\left(\left[Z_{2}, X_{j}\right], \phi X_{i}\right)+2 g\left(X_{j}, X_{i}\right) \\
& =g\left(\nabla_{Z_{2}} X_{j}-\nabla_{X_{j}} Z_{2}, \phi X_{i}\right)+2 g\left(X_{j}, X_{i}\right) \\
& =-g\left(X_{j}, \nabla_{Z_{2}} \phi X_{i}\right)+g\left(Z_{2}, \nabla_{X_{j}} \phi X_{i}\right)+2 g\left(X_{j}, X_{i}\right) \\
& =g\left(Z_{2}, \nabla_{X_{i}} \phi X_{j}\right)+2 g\left(X_{j}, X_{i}\right) \\
& =g\left(Z_{2},\left(\nabla_{X_{i}} \phi\right) X_{j}\right)+2 g\left(X_{j}, X_{i}\right)
\end{aligned}
$$

which gives

$$
g\left(\left(\nabla_{X_{i}} \phi\right) X_{j}, Z_{2}\right)=2 g\left(X_{i}, X_{j}\right) .
$$

Using (43) we obtain:

$$
\begin{aligned}
g\left(\left(\nabla_{X_{i}} \phi\right) X_{j}, Z_{1}\right) & =g\left(\nabla_{X_{i}} \phi X_{j}, Z_{1}\right) \\
& =-g\left(\phi X_{j}, \nabla_{X_{i}} Z_{1}\right) \\
& =-g\left(\phi X_{j}, \nabla_{X_{i}} Z\right)+g\left(\phi X_{j}, \nabla_{X_{i}} Z_{2}\right) \\
& =-g\left(\phi X_{j},-2 \phi X_{i}\right)-g\left(\nabla_{X_{i}} \phi X_{j}, Z_{2}\right) \\
& =2 g\left(X_{j}, X_{i}\right)-g\left(\left(\nabla_{X_{i}} \phi\right) X_{j}, Z_{2}\right) \\
& =0
\end{aligned}
$$


Equations (43), (44) and Corollary 4.8 then give for every $X, Y \in[+1]_{2}$

$$
\left(\nabla_{X} \phi\right) Y=2 g(X, Y) Z_{2}
$$

Moreover (32) implies $\left[X_{i}, X_{j}\right]=0$ and then $[+1]_{2}$ is integrable. With a similar argument we see that $[+1]_{1}$ is also integrable.

g) Set $\dot{h}_{2}=\frac{1}{2} \mathcal{L}_{Z_{2}} \phi_{2}$, let $L$ be a leaf of $T \mathcal{F}_{1}$ considered as a submanifold and $X$ tangent to $L$. Let $\dot{\nabla}$ be the induced connection, $\sigma$ the second fundamental form, $A_{Z_{1}}$ the Weingarten operator in the direction $Z_{1}$ and $\nabla^{\prime}$ the connection in the normal bundle. Then we have:

$$
\begin{gathered}
\nabla_{X} Z=-\phi X-\phi \mathrm{h} X=-\phi_{2} X-\phi_{2} \mathrm{~h}_{2} X \\
\nabla_{X} Z=\nabla_{X} Z_{1}+\nabla_{X} Z_{2}=-A_{Z_{1}} X+\nabla_{X}^{\prime} Z_{1}+\dot{\nabla}_{X} Z_{2}+\sigma\left(X, Z_{2}\right) .
\end{gathered}
$$

Comparing the previous equations we obtain

$$
\begin{gathered}
A_{Z_{1}} X=\phi_{2}\left(\mathrm{~h}_{2} X-\dot{\mathrm{h}}_{2} X\right)=\phi\left(\mathrm{h} X-\dot{\mathrm{h}}_{2} X\right) \\
\nabla_{X}^{\prime} Z_{1}=-\sigma\left(X, Z_{2}\right) .
\end{gathered}
$$

Moreover it is clear that we have $\sigma\left(Z_{2}, Z_{2}\right)=0$ and $A_{Z_{1}} Z_{2}=0$.

We want to prove that $\mathrm{h}_{2}=\dot{\mathrm{h}}_{2}$ and this is equivalent to proving the vanishing of $A_{Z_{1}}$. To do this we will prove the vanishing of $g\left(A_{Z_{1}} X, Y\right)$ for $X, Y$ elements of the eigenbasis of $\mathrm{h}$ constructed before.

First observe that

$$
g\left(A_{Z_{1}} X, Z_{2}\right)=g\left(\phi\left(\mathrm{h} X-\dot{\mathrm{h}}_{2} X\right), Z_{2}\right)=0,
$$

and then we have to prove our statement for $X, Y$ horizontal. Also note that, by direct calculation, we have:

$$
g\left(A_{Z_{1}} \phi X, Y\right)=g\left(A_{Z_{1}} X, \phi Y\right) .
$$

Then it remains to prove that $g\left(A_{Z_{1}} X_{i}, X_{j}\right)$ and $g\left(A_{Z_{1}} \phi X_{i}, X_{j}\right)$ both vanish.

For the first one:

$$
\begin{aligned}
g\left(A_{Z_{1}} X_{i}, X_{j}\right) & =g\left(\phi\left(\mathrm{h} X_{i}-\dot{\mathrm{h}}_{2} X_{i}\right), X_{j}\right) \\
& =g\left(\phi\left(X_{i}-\dot{\mathrm{h}}_{2} X_{i}\right), X_{j}\right) \\
& =g\left(\dot{\mathrm{h}}_{2} X_{i}, \phi X_{j}\right) \\
& =\frac{1}{2}\left(g\left(\left[Z_{2}, \phi_{2} X_{i}\right]-\phi_{2}\left[Z_{2}, X_{i}\right], \phi_{2} X_{j}\right)\right) \\
& =\frac{1}{2}\left(g\left(\nabla_{Z_{2}} \phi_{2} X_{i}-\nabla_{\phi_{2} X_{i}} Z_{2}, \phi_{2} X_{j}\right)-g\left(\phi_{2}\left[Z_{2}, X_{i}\right], \phi_{2} X_{j}\right)\right) \\
& =\frac{1}{2}\left(g\left(\nabla_{Z_{2}} \phi_{2} X_{i}, \phi_{2} X_{j}\right)-g\left(\left[Z_{2}, X_{i}\right], X_{j}\right)\right) \\
& =0
\end{aligned}
$$

because $\left[Z_{2}, X_{i}\right]$ is in $[-1]_{2}+\mathbb{R} Z_{2}$ and where we have used (26) and (27). 
Now we have:

$$
\begin{aligned}
g\left(A_{Z_{1}} X_{i}, \phi X_{j}\right) & =g\left(\phi_{2}\left(\mathrm{~h}_{2} X_{i}-\dot{\mathrm{h}}_{2} X_{i}\right), \phi_{2} X_{j}\right) \\
& =g\left(X_{i}-\dot{\mathrm{h}}_{2} X_{i}, X_{j}\right) \\
& =g\left(X_{i}, X_{j}\right)-g\left(\dot{\mathrm{h}}_{2} X_{i}, X_{j}\right) \\
& =g\left(X_{i}, X_{j}\right)-\frac{1}{2} g\left(\left[Z_{2}, \phi_{2} X_{i}\right]-\phi_{2}\left[Z_{2}, X_{i}\right], X_{j}\right) \\
& \left.=g\left(X_{i}, X_{j}\right)-\frac{1}{2} g\left(\left[Z_{2}, X_{i}\right], \phi_{2} X_{j}\right)\right) \\
& \left.=g\left(X_{i}, X_{j}\right)-\frac{1}{2} g\left(\nabla_{Z_{2}} X_{i}-\nabla_{X_{i}} Z_{2}, \phi_{2} X_{j}\right)\right) \\
& =g\left(X_{i}, X_{j}\right)-\frac{1}{2}\left(-g\left(X_{i}, \nabla_{Z_{2}} \phi_{2} X_{j}\right)+g\left(Z_{2},\left(\nabla_{X_{i}} \phi_{2}\right) X_{j}\right)\right) \\
& =0,
\end{aligned}
$$

where at the end we used (26) and (45).

h) To prove that $[+1]_{2}$ is also totally geodesic, let us consider the operators $H_{i}=\frac{1}{2} \mathcal{L}_{Z_{i}} \phi$. Each $H_{i}$ is symmetric by Theorem 3.4. From our observation above that $\mathrm{h}_{2}=\dot{\mathrm{h}}_{2}$ a simple direct computation shows that we have

$$
H_{2} X_{i}=X_{i} \text { and similarly } H_{1} Y_{r}=Y_{r} \text {. }
$$

This implies that $H_{1} X_{i}$ has no $Y_{r}$ or $\phi Y_{r}$ component. Thus since $A_{Z_{1}}$ vanishes we have

$$
H_{1} X_{i}=0 \text { and similarly } H_{2} Y_{i}=0 .
$$

Applying (45), we obtain

$$
2 g\left(X_{i}, X_{j}\right) Z_{2}=\nabla_{X_{i}} \phi X_{j}-\phi \nabla_{X_{i}} X_{j},
$$

which, differentiating along $Y_{r}$, gives:

$$
2\left(Y_{r} g\left(X_{i}, X_{j}\right)\right) Z_{2}+2 g\left(X_{i}, X_{j}\right) \nabla_{Y_{r}} Z_{2}=\nabla_{Y_{r}} \nabla_{X_{i}} \phi X_{j}-\left(\nabla_{Y_{r}} \phi\right) \nabla_{X_{i}} X_{j}-\phi \nabla_{Y_{r}} \nabla_{X_{i}} X_{j} .
$$

Taking the scalar product with $Z_{2}$, we get

$$
2 Y_{r} g\left(X_{i}, X_{j}\right)=g\left(\nabla_{Y_{r}} \nabla_{X_{i}} \phi X_{j}, Z_{2}\right)-g\left(\left(\nabla_{Y_{r}} \phi\right) \nabla_{X_{i}} X_{j}, Z_{2}\right) .
$$

Now, applying (4), we obtain

$$
\begin{aligned}
-g\left(\left(\nabla_{Y_{r}} \phi\right) \nabla_{X_{i}} X_{j}, Z_{2}\right) & =g\left(\nabla_{X_{i}} X_{j},\left(\nabla_{Y_{r}} \phi\right) Z_{2}\right) \\
& =-g\left(\phi H_{2} \nabla_{X_{i}} X_{j}, \phi Y_{r}\right)-d \alpha_{2}\left(\phi \nabla_{X_{i}} X_{j}, Y_{r}\right) \\
& =-g\left(H_{2} \nabla_{X_{i}} X_{j}, Y_{r}\right) \\
& =-g\left(\nabla_{X_{i}} X_{j}, H_{2} Y_{r}\right) \\
& =0 .
\end{aligned}
$$

Applying (41) again we first note that by computing as we have been doing, the above properties of $H_{1}$ and $H_{2}$ yield

$$
g\left(\left(\nabla_{Y_{r}} \phi\right) X_{j}, Z_{1}\right)=\underset{20}{g}\left(\left(\nabla_{Y_{r}} \phi\right) X_{j}, Z_{2}\right)=0
$$


Corollary 4.8 then gives $\left(\nabla_{Y_{r}} \phi\right) X_{j}=0$ and therefore by (4)

$$
\begin{aligned}
g\left(\nabla_{X_{i}} \nabla_{Y_{r}} \phi X_{j}, Z_{2}\right) & =g\left(\left(\nabla_{X_{i}} \phi\right) \nabla_{Y_{r}} X_{j}, Z_{2}\right) \\
& =+g\left(H_{2} \nabla_{Y_{r}} X_{j}, X_{i}\right)+d \alpha_{2}\left(\phi \nabla_{Y_{r}} X_{j}, X_{i}\right) \\
& =2 g\left(\nabla_{Y_{r}} X_{j}, X_{i}\right) .
\end{aligned}
$$

Equation (49) then becomes

$$
\begin{aligned}
2 Y_{r} g\left(X_{i}, X_{j}\right) & =g\left(\nabla_{Y_{r}} \nabla_{X_{i}} \phi X_{j}, Z_{2}\right) \\
& =g\left(R_{Y_{r} X_{i}} \phi X_{j}, Z_{2}\right)+g\left(\nabla_{X_{i}} \nabla_{Y_{r}} \phi X_{j}, Z_{2}\right) \\
& =2 g\left(\nabla_{Y_{r}} X_{j}, X_{i}\right)
\end{aligned}
$$

since $\left[Y_{r}, X_{j}\right]=0$ by (32). But, by the compatibility condition of the Levi-Civita connection we also have

$$
Y_{r} g\left(X_{i}, X_{j}\right)=g\left(\nabla_{Y_{r}} X_{i}, X_{j}\right)+g\left(X_{i}, \nabla_{Y_{r}} X_{j}\right) .
$$

Comparing this with (152), gives

$$
g\left(\nabla_{Y_{r}} X_{i}, X_{j}\right)=0
$$

and in turn, again noting $\left[Y_{r}, X_{j}\right]=0$,

$$
g\left(Y_{r}, \nabla_{X_{j}} X_{i}\right)=0
$$

Since $[+1]$ is totally geodesic, we also have $g\left(\nabla_{X_{j}} X_{i}, \phi Y_{r}\right)=0$, therefore $[+1]_{2}$ is totally geodesic.

i) We rewrite (45) as follows

$$
2 g\left(X_{i}, X_{j}\right) Z_{2}=\left(\nabla_{X_{i}} \phi\right) X_{j}=\nabla_{X_{i}} \phi X_{j}-\phi \nabla_{X_{i}} X_{j},
$$

and we want to apply $\nabla_{X_{l}}$ to (53). We firstly need the following calculations:

$$
\begin{aligned}
2 g\left(X_{i}, X_{j}\right) & =g\left(\left(\nabla_{X_{i}} \phi\right) X_{j}, Z_{2}\right) \\
& =-g\left(X_{j},\left(\nabla_{X_{i}} \phi\right) Z_{2}\right) \\
& =-g\left(X_{j},-\phi\left(\nabla_{X_{i}} Z_{2}\right)\right) \\
& =-g\left(\phi X_{j}, \nabla_{X_{i}} Z_{2}\right) .
\end{aligned}
$$

and therefore

$$
g\left(\nabla_{X_{l}} Z_{2}, \phi X_{m}\right)=-2 g\left(X_{l}, X_{m}\right) .
$$

Applying $\nabla_{X_{l}}$ to (53) we obtain

$$
\begin{array}{r}
2\left(X_{l} g\left(X_{i}, X_{j}\right)\right) Z_{2}+2 g\left(X_{i}, X_{j}\right) \nabla_{X_{l}} Z_{2}= \\
\nabla_{X_{l}} \nabla_{X_{i}} \phi X_{j}-\left(\nabla_{X_{l}} \phi\right)\left(\nabla_{X_{i}} X_{j}\right)-\phi\left(\nabla_{X_{l}} \nabla_{X_{i}} X_{j}\right) .
\end{array}
$$

Taking the scalar product with $\phi X_{m}$ we get

$$
-4 g\left(X_{i}, X_{j}\right) g\left(X_{l}, X_{m}\right)=g\left(\nabla_{X_{l}} \nabla_{X_{i}} \phi X_{j}-\phi\left(\nabla_{X_{l}} \nabla_{X_{i}} X_{j}\right), \phi X_{m}\right),
$$

since $g\left(\left(\nabla_{X_{l}} \phi\right)\left(\nabla_{X_{i}} X_{j}\right), \phi X_{m}\right)=0$ by Corollary 4.8 . 
j) Note that from Theorem 2.9 we know that the integral submanifolds of $\mathcal{V}$ are totally geodesic. Moreover we proved that $[+1]_{1}$ and $[+1]_{2}$ are integrable and totally geodesic. We also know that $[-1]_{1}$ and $[-1]_{2}$ are integrable, totally geodesic and flat by (27) and (28). Then the manifold $M$ splits locally as a Riemannian product of integrable submanifolds of $\mathcal{V},[ \pm 1]_{1}$ and $[ \pm 1]_{2}$. To conclude the proof we exhibit the curvature. To do this interchange the role of $X_{l}$ and $X_{i}$ in (55) and take the difference. Then we have

$$
-4 g\left(X_{i}, X_{j}\right) g\left(X_{l}, X_{m}\right)+4 g\left(X_{l}, X_{j}\right) g\left(X_{i}, X_{m}\right)=g\left(R_{X_{l} X_{i}} \phi X_{j}, \phi X_{m}\right)-g\left(R_{X_{l} X_{i}} X_{j}, X_{m}\right) .
$$

The first term on the right vanishes by the local Riemannian product structure and the second term then gives the desired value of the curvature. If $h$ or $k$ is equal to 1 , the corresponding $[+1]_{i}$ subbundle is 1 -dimensional and the leaves of the characteristic foliation are flat.

Corollary 5.2. Let $M$ be a $(2 h+2 k+2)$-dimensional manifold endowed with a metric contact pair $\left(\alpha_{1}, \alpha_{2}, \phi, g\right)$ of type $(h, k)$ (with $h \geq 1$ ) and decomposable $\phi$, and such that the curvature of $g$ vanishes on the vertical subbundle. If $M$ is complete, then its Riemannian universal covering is isometric to $\mathbb{E}^{h+1} \times \mathbb{S}^{h}(4) \times \mathbb{E}^{k+1} \times \mathbb{S}^{k}(4)$ if $k \geq 1$ or $\mathbb{E}^{h+1} \times \mathbb{S}^{h}(4) \times \mathbb{E}^{1}$ if $k=0$. It is to be understood that when $h$ (or $k$ ) is equal to 1 , the $\mathbb{S}^{h}(4)$ factor will just contribute another line to the Euclidean factor.

Proof. The Riemannian universal covering $\tilde{M}$ is locally isometric to $M$ and then by Theorem 5.1 is locally isometric to $\mathbb{E}^{h+1} \times \mathbb{S}^{h}(4) \times \mathbb{E}^{k+1} \times \mathbb{S}^{k}(4)$ if $k \geq 1$ or to $\mathbb{E}^{h+1} \times \mathbb{S}^{h}(4) \times \mathbb{E}^{1}$ if $k=0$. Then one concludes by applying the de Rham Decomposition Theorem.

Corollary 5.3. Let $M$ be a $(2 h+2 k+2)$-dimensional manifold endowed with a metric contact pair $\left(\alpha_{1}, \alpha_{2}, \phi, g\right)$ of type $(h, k)$ (with $\left.h \geq 1\right)$ and decomposable $\phi$. If $g$ is flat then $h, k \leq 1$.

\section{REFERENCES}

1. K. Abe, On a class of Hermitian manifolds, Invent. Math. 51 (1979), 103-121.

2. G. Bande, Formes de contact généralisé, couples de contact et couples contacto-symplectiques, Thèse de Doctorat, Université de Haute Alsace, Mulhouse, 2000.

3. G. Bande, Couples contacto-symplectiques, Trans. Amer. Math. Soc. 355 (2003), 1699-1711.

4. G. Bande, P. Ghiggini and D. Kotschick, Stability theorems for symplectic and contact pairs, Int. Math. Res. Not. 68 (2004), 3673-3688.

5. G. Bande and A. Hadjar, Contact Pairs, Tohoku Math. J. 57 (2005), 247-260.

6. G. Bande and A. Hadjar, Contact pair structures and associated metrics, Differential Geometry Proceedings of the 8th International Colloquium, World Sci. Publ. (2009), 266-275.

7. G. Bande and A. Hadjar, On normal contact pairs, Internat. J. Math. 21 (2010), 737-754.

8. G. Bande and A. Hadjar, On the characteristic foliations of metric contact pairs, Harmonic maps and differential geometry, 255-259, Contemp. Math., 542, Amer. Math. Soc., Providence, RI, 2011.

9. G. Bande and D. Kotschick, The Geometry of Symplectic pairs, Trans. Amer. Math. Soc. 358 (2006), $1643-1655$.

10. G. Bande and D. Kotschick, Contact pairs and locally conformally symplectic structures, Harmonic maps and differential geometry, 85-98, Contemp. Math., 542, Amer. Math. Soc., Providence, RI, 2011..

11. D. E. Blair, On the non-existence of flat contact metric structures, Tohoku Math. J. 28 (1976), 373-379.

12. D. E. Blair, Two remarks on contact metric structures, Tohoku Math. J. 29 (1977), 319-324.

13. D. E. Blair, Riemannian geometry of contact and symplectic manifolds, Progress in Mathematics, 2nd Ed., vol. 203, Birkhäuser, 2010. 
14. D. E. Blair, G. D. Ludden and K. Yano, Geometry of complex manifolds similar to the Calabi-Eckmann manifolds, J. Differential Geom. 9 (1974), 263-274.

15. A. Borel, Compact Clifford-Klein forms of symmetric spaces, Topology 2 (1963), 111-122.

16. S. Dragomir and L. Ornea, Locally conformal Kähler geometry, Progress in Mathematics, vol. 155, Birkhäuser, 1998.

17. H. Geiges, Normal contact structures on 3-manifolds, Tohoku Math. J. 49 (1997), 415-422.

18. Y. Hatakeyama, Y. Ogawa and S. Tanno, Some properties of manifolds with contact metric structure, Tohoku Math. J. 15 (1963), 42-48.

19. I. Vaisman, Some curvature properties of locally conformal Kähler manifolds, Trans. Amer. Math. Soc. 259 (1980), 439-447.

Dipartimento di Matematica e Informatica, Università degli studi di Cagliari, Via OsPEDAle 72, 09124 Cagliari, Italy

E-mail address: gbande@unica.it

Department of Mathematics, Michigan State University, East Lansing, Mi 48824-1027, USA

E-mail address: blair@math.msu.edu

Laboratoire de Mathématiques, Informatique et Applications, Université de Haute AlSaCe - 4, Rue de Frères Lumière, 68093 Mulhouse Cedex, France

E-mail address: mohamed.hadjar@uha.fr 